\title{
A NON-STANDARD TRUTH DEFINITION
}

\section{ROBERT MCNAUGHTON ${ }^{1}$}

1. Introduction. Wang has shown in [1] that in a certain system $Z_{C}$ the impredicative axiom schema of set existence cannot be reduced to finitely many special axioms, provided $Z_{C}$ is consistent. $\mathrm{He}$ shows that, if $Z_{C}$ is consistent, any system $Z_{C}^{\prime}$ containing only a finite number of axioms of $Z_{C}$ has a model $D^{\prime}$ containing only denumerably many sets. The enumeration of these sets can actually be given in $Z_{C}$, and, by the diagonal method, a set is constructed in $Z_{C}$ which cannot be in $D^{\prime}$. Hence it is impossible to prove the existence of the set in $Z_{C}^{\prime}$. ( $Z_{C}$ is an extension of the Zermelo-Fraenkel set theory. In this connection, cf. [3].)

In this paper, I shall make use of Wang's enumeration of the sets of the model $D^{\prime}$ in $Z_{C}$, and go on to define a truth predicate in $Z_{C}$ for $D^{\prime}$. Let $F(m)$ be the formula whose Gödel number is $m$. Then the truth predicate is ' $\operatorname{Tr}(m)$ ' which means that $F(m)$ is true in the model $D^{\prime}$. Since $D^{\prime}$ is a non-standard model (in the sense of [4]), I shall call this a non-standard truth predicate. I shall prove the consistency of $Z_{C}^{\prime}$ in $Z_{C}$, using this predicate.

This non-standard truth definition may seem strange, since the literature is concerned mostly with standard truth definitions. But there is reason to be interested in non-standard truth definitions as well as standard ones. For if there is no such thing as absolute mathematical truth, as many mathematicians and philosophers are now inclined to believe, then it would seem that the notion of truth has to be relative to a model. (Indeed, in [5] Rosser and Wang express their willingness to work with systems demonstrably without any standard models.) A mathematical statement true in one model may be false in another; and if to one of these models we attribute a kind of pre-eminence, it is only because we are more familiar with it. We should not say, then, that a certain mathematical statement is true; rather we should say that it is true in such and such model.

Following [6] the usual manner of constructing a truth definition for a system $P$ involves the devices of another system $P^{\prime}$ whose notation is richer than $P$. E.g., $P^{\prime}$ may contain a new type of variable. Also such truth definitions are associated with a standard concept of truth. Thus the theorems provable in $P^{\prime}$, answering to the criterion of

Received by the editors April 18, 1953 and, in revised form, October 19, 1953.

1 The result of this paper was obtained while I was doing research at Illinois Institute of Technology sponsored by the Office of Naval Research. 
adequacy for the truth definition, are of the form

$$
{ }^{\top} \operatorname{Tr}(\bar{m}) \equiv F(\bar{m})^{1},
$$

where $F(\bar{m})$ has no free variables. (The corners here are as in [7] for quasi-quotation. ' $\bar{m}$ ' or any letter with an upper bar stands ambiguously for a numeral.) However, (1) cannot in general be proved in $Z_{C}$. For the non-standard model $D^{\prime}$ is not a model for $Z_{C}$.

However, although (1) does not hold for the truth definition of this paper, something analogous does hold. For a formula $F(\bar{m})$ another formula $F(\operatorname{trans}(\bar{m})$ ) will be constructed in $\S 3$, not provable in $Z_{C}$ unless $F(\bar{m})$ is true in $D^{\prime}$. Then, in $Z_{C}$ it is possible to prove theorems of the following form:

$$
{ }^{\top} \operatorname{Tr}(\bar{m}) \equiv F(\operatorname{trans}(\bar{m})){ }^{\top},
$$

where $F(\bar{m})$ and $F($ trans $(\bar{m}))$ have no free variables. These theorems answer to a more general criterion of adequacy for a truth definition, in fact to a criterion originally proposed by Tarski. (Cf. p. 305 of [6].)

The non-standard truth definition is similar to what is called in [8] a "model of the second kind." The consistency proof of this paper is similar to the consistency proof sketched in $\$ 4$ of [8].

2. The truth definition. I shall use terminology of [1]. On p. 61 of that paper a set $e$ is defined which presents the enumeration of the sets of $D^{\prime} . e(0), e(1), e(2), \cdots$ are all the sets of $D^{\prime}$. (Arrows above the $e$ 's are omitted because of printing difficulties.) In order to define truth in $D^{\prime}$, it is first necessary (following [6] and [2]) to define a notion of satisfaction; a formula is satisfied in $D^{\prime}$ by an assignment of its free variables as entities of $D^{\prime}$ if it is true with its free variables so interpreted. We can assume that ' $x_{1}$ ', ' $x_{2}$ ', $\cdots$ are all the variables of $Z_{C}^{\prime}$ in primitive notation. Letters of the middle of the alphabet (' $g$ ' to ' $t$ ') are the variables in defined notation for natural numbers. A natural number $g$ represents an assignment of the variables to sets of $D^{\prime}$ as follows: for $g>0, g_{i}$ is the exponent of the $(i+1)$ th prime number in the prime factorization of $g$; we assume that $0_{i}=1_{i}=0$; then $g$ assigns $e\left(g_{i}\right)$ to the variable ' $x_{i}$ ', for every $i$. For any formula, as $g$ goes through all positive integers, every possible set of interpretations of its free variables as sets of $D^{\prime}$ will be assured, since the formula can have only a finite number of free variables.

The satisfaction predicate ' $g S m$ ', meaning that assignment $g$ satisfies $F(m)$ in $D^{\prime}$, can be defined after a few observations. It is known that all formulas can be built up from the atomic formulas by means of the Sheffer stroke function and universal quantification. It is 
known that the following are definable in $Z_{C}$ (since $Z_{C}$ contains number theory): ' $M(m, n, k)$ ', meaning that $F(m)$ is ' $x_{n} \in x_{k}$ '; ' $T(m, n, k)$ ' meaning that $F(m)$ is ${ }^{\top} F(n) \mid F(k)^{\top}$ (the Sheffer stroke function); ' $Q(m, n, k)$ ' meaning that $F(m)$ is ${ }^{\top}\left(x_{n}\right) F(k)^{\top}$ (universal quantification).

Let $R_{m}$ be the number of logical operators in $F(m)$. A logical operator is either a stroke function or a universal quantification. It will be necessary to be able to change the assignment $g$ to an alternate assignment $t(g, i, m)$ such that $(t(g, i, m))_{i}$ is $m$ and, for $j \neq i$, $(t(g, i, m))_{j}=g_{j}$. Clearly ' $t(g, i, m)$ ' is definable in $Z_{C}$. ' $G_{i}(y)$ ' means that $y$ is an $i$-satisfaction set; in other words, $y$ is the set of all ordered pairs $\langle g, m\rangle$ such that $R_{m} \leqq i$ and $g$ satisfies $F(m)$ in $D^{\prime}$. Formally, ' $G_{i}(y)$ ' is an abbreviation for the following:

$$
\begin{aligned}
\langle g, m\rangle \in & y . \equiv . R_{m} \leqq i .(\exists n)(\exists k) M(m, n, k) . e\left(g_{n}\right) \\
& \in e\left(g_{k}\right) . \vee . T(m, n, k) \cdot\langle g, n\rangle \in y \mid\langle g, k\rangle \in y \cdot \mathrm{V} \cdot Q(m, n, k) \\
& \cdot(j)(\langle t(g, n, j), k\rangle \in y)) .
\end{aligned}
$$

' $g S m^{\prime}$ ' ( $g$ satisfies $F(m)$ ) and ' $\operatorname{Tr}(m)$ ' $(F(m)$ is true) are defined, respectively, as follows:

$$
\begin{aligned}
& (\exists y)\left(G_{R_{m}}(y) \cdot\langle g, m\rangle \in y\right), \\
& (g) g S m .
\end{aligned}
$$

3. The basic theorems for the truth definition. This section culminates in a proof of the fact that, for every numeral ' $\bar{m}$ ' such that $F(\bar{m})$ has no free variables, ${ }^{[} \operatorname{Tr}(\bar{m}) \equiv F(\text { trans }(\bar{m}))^{\top}$ is a theorem of $Z_{C}$. This proof justifies the truth definition.

In this section and the following section a number of theorems, which are formal theorems of $Z_{C}$, are proved; because these theorems are similar to certain formal theorems of the second section of [2], the proofs in the present paper are just sketched. Familiarity with [2] (to which all references are henceforth made) is therefore assumed on the part of the reader, who will also note that the development of the truth definition of [2] is similar to the development in this paper, except for two differences. First, the truth definition of [2] is a standard one and therefore requires a higher type variable, namely, ' $X_{1}$ ', ' $X_{2}$ ', etc. Second, while $g$ in this paper is a natural number, $g$ in [2] is a certain kind of set; however, $g$ represents a sequence, in [2] as well as in this paper.

THEOREM I. (i) $(\exists y) G_{i}(y)$.

The proof is similar to the proof of 2.33 (p. 250) including the 
proofs of Lemmas 2.30 and 2.31. Here the axiom of separation of $Z_{C}$ is used instead of the class axiom of [2].

THEOREM II.

(1) $M(m, n, k) \supset . g S m \equiv e\left(g_{n}\right) \in e\left(g_{k}\right)$.

(2) $T(m, n, k) \supset: g S m \equiv . g S n \mid g S k$.

(3) $Q(m, n, k) \supset . g S m \equiv(j) t(g, n, j) S k$.

The proofs of (1), (2), (3) are precisely similar to the proofs of $2.40,2.41$, and 2.42 (p. 251). Note that corresponding theorems can be proved in $Z_{C}$ involving different logical operators; for example, where ' $\supset(m, n, k)$ ' means that $F(m)$ is ' $F(n) \supset F(k)$ ', the following: $\supset(m, n, k) \supset: g S m \equiv . g S n \supset g S k$. (Cf. 4.1, p. 258.)

The translation of statements of $Z_{C}^{\prime}$ into statements of $Z_{C}$ about the model $D^{\prime}$ is now given (following [1]). Let us assume that ' $m_{1}$ ', ' $m_{2}$ ', etc. are infinitely many variables in $Z_{C}$, in defined notation, for the natural numbers. Then $F(\operatorname{trans}(m))$ is obtained from $F(m)$, assuming that $F(m)$ is in primitive notation, by replacing each variable ' $x_{i}^{\prime}$ ' by ' $e\left(m_{i}\right)$ ' in all context except within quantifiers. ' $x_{i}$ ' within quantifiers is replaced by ' $m_{i}$ '. For example, ' $\left(x_{i}\right)\left(x_{i} \in x_{i}\right)$ ' is translated as ' $\left(m_{i}\right)\left(e\left(m_{i}\right) \in e\left(m_{i}\right)\right)$ '. Clearly 'trans $(m)$ ', being recursive, is definable in number theory and hence in $Z_{C}$. As is mentioned in [1], where $F(m)$ is provable in $Z_{C^{\prime}}^{\prime}, F(\operatorname{trans}(m))$ is provable in $Z_{C}$.

Theorem III. For any numeral ' $\bar{m}$ ' such that $F(\bar{m})$ has no free variables, the following is a theorem of $\left.Z_{C}:{ }^{\top} \operatorname{Tr}(\bar{m})\right) \equiv F(\operatorname{trans}(\bar{m}))$.

The proof is similar to the proof of 2.44 (p. 252) including the proof of Lemma 2.43. There is a dependency here on Theorem II, similar to the dependency in [2] on $2.40,2.41$, and 2.42 .

4. The consistency proof. Let ' $A x(m)$ ' (' $T h m(m)$ ') mean that $F(m)$ is an axiom (theorem) of $Z_{C}^{\prime}$. It is now convenient to refer to $\S 4$ of $[2]$.

Theorem IV. $(m)(A x(m) \supset \operatorname{Tr}(m))$.

The proof is similar to the proof of 4.8 (p. 259) including the proof of Lemma 4.7 (p. 258). The notion ' $a x f r(m)$ ' used in 4.7 is easily definable in $Z_{C}$. We must note also that impredicative features exist in $Z_{c}$, corresponding to those used in [2].

Theorem V. $(m)(\operatorname{Thm}(m) \supset \operatorname{Tr}(m))$.

The proof is similar to the proof of 4.12 (p. 259), including the proofs of 4.10 and 4.11 . 
From this it follows readily that the consistency of $Z_{C^{\prime}}$ is provable in $Z_{C}$. (Cf. Theorem IV, p. 260, of [2].)

\section{BIBLIOGRAPHY}

1. Hao Wang, The irreducibility of impredicative principles, Math. Ann. vol. 125 (1952) pp. 56-66.

2. - Truth definitions and consistency proofs, Trans. Amer. Math. Soc. vol. 73 (1952) pp. 243-275.

3. R. McNaughton, Review of [1], J. Symbolic Logic vol. 18 (1953) pp. 265-266.

4. L. Henkin, Completeness in the theory of types, J. Symbolic Logic vol. 15 (1950) pp. 81-91.

5. B. Rosser and Hao Wang, Non-standard models for formal logics, J. Symbolic Logic vol. 15 (1950) pp. 113-129.

6. A. Tarski, Der Wahrheitsbegriff in den formalisierten Sprachen, Studia Philosophica vol. 1 (1936) pp. 251-405.

7. W. V. Quine, Mathematical logic, New York, 1940.

8. A. Mostowski, On models of axiomatic systems, Fund. Math. vol. 39 (1952) pp. 133-158.

ILLINOIS INSTITUTE OF TECHNOLOGY

\section{AN EXTENSION THEOREM FOR SOLUTIONS OF $d \omega=\Omega$}

\section{HARLEY FLANDERS}

Let $U$ and $V$ be open sets in $E_{n}$ such that $\bar{V} \subset U$ and $U$ is connected and homologically trivial, i.e., all homology groups of $U$ beyond the zero-dimensional case vanish. Let $\Omega$ be an exterior differential form of degree $p$ on $E_{n}$ with infinitely differentiable coefficients whose exterior derivative vanishes: $d \Omega=0$. The well known converse to the Lemma of Poincare asserts that $\Omega=d \omega$ where $\omega$ is an infinitely differentiable $p-1$ form on $E_{n}$. Let us suppose however that we are merely given a $p-1$ form $\alpha$ on $U$ such that $d \alpha=\Omega$ on $U$. The question immediately arises as to whether it is possible to prolong $\alpha$ to all of $E_{n}$. The example $U=\{(x, y) \mid x>0\}, \alpha=(x d y-y d x) /\left(x^{2}+y^{2}\right)$, $\Omega=0$ shows us that the answer is negative. Nevertheless, there exists $a p-1$ form $\beta$ on $E_{n}$ such that $\beta=\alpha$ on $V$ and $d \beta=\Omega$ on $E_{n}$.

To prove this, we shall take for granted the existence of an infinitely differentiable function $f$ on $E_{n}$ such that $f=1$ on $V$ and $f=0$ outside of a closed subset of $U$. We have the form $\omega$ on $E_{n}$ such that $d \omega=\Omega$ on $E_{n}$ and the form $\alpha$ on $U$ such that $d \alpha=\Omega$ on $U$. Thus $d(\alpha-\omega)=0$ on $U$ and so it follows from the hypotheses on $U$ and what is essentially de Rham's second theorem that $\alpha-\omega=d \lambda$ on $U$,

Presented to the Society, November 28, 1953; received by the editors August 31, 1953 and, in revised form, October 5, 1953. 